\title{
EFFICACY OF YOGA IN INDIVIDUALS WITH FIBROMYALGA: A SYSTEMATIC REVIEW
}

\author{
Isabela Melo Froes Ribeiro ${ }^{1, \star}$, Susan Lee King Yuan¹, Amélia Pasqual Marques \\ 1.Universidade de São Paulo, São Paulo (SP), Brazil. \\ *Corresponding author: isabelafroes15@gmail.com
}

\section{BACKGROUND}

Yoga is a practical-philosophical system that incorporates postures, body movements, breathing techniques and meditation. Clinical trials have been investigating the efficacy of this practice in fibromyalgia. The objective of this review was to identify, appraise and synthesize all available evidence on the efficacy of yoga in fibromyalgia.

\section{METHODS}

An electronic search was conducted at SciELO, LILACS, MEDLINE, Scopus, Web of Science, Embase and Cochrane CENTRAL (Jan 1990-Aug 2020). We selected randomized trials which included participants diagnosed with fibromyalgia, used yoga as the primary intervention, and assessed the following outcomes: quality of life (QOL), pain, fatigue, quality of sleep and/or psychological distress. Two independent reviewers screened records and examined full-text reports for compliance with the eligibility criteria. One of the reviewers performed the data extraction and risk of bias assessment using the PEDro scale, and the other checked. A qualitative analysis of the data was performed.

\section{RESULTS AND CONCLUSION}

The search identified 1364 records. After removing duplicates, 1,006 records were screened. Fifty full-text reports were assessed for eligibility and six studies were included. A study of good methodological quality from 2010 reported immediate benefits of yoga on QOL, pain, fatigue, anxiety, depression and psychological distress in participants with fibromyalgia, compared with an inactive control. No statistically significant effect was observed in the quality of sleep. In a low-quality study from 2007), the use of tui na as a complementary therapy to yoga was not beneficial for QOL or pain. A good-quality study from 2008 found that the use of underwater yoga breathing as a complementary therapy to recreational activities was beneficial for QOL, fatigue and quality of sleep. There were inconsistencies in the results for anxiety and no effects were observed for depression. A moderate-quality study from 2007 and a good-quality one from 2011 compared mindfulness-based stress reduction (MBSR) to an inactive control, and found no effects on QOL, pain and quality of sleep. Possibly, it has an effect on anxiety, but there were inconsistencies in the results for depression. The latter study reported that MBSR was not superior to a conventional program of relaxation and stretching exercises regarding QOL, pain, quality of sleep, anxiety and depression. A moderate-quality study from 2003 found that MBSR + qigong was not superior to a conventional educational intervention regarding QOL, pain and depression.

\section{KEYWORDS:}

Fibromyalgia, Yoga, Mindfulness, Quality of life, Systematic review. 